\title{
Anemia crónica grave por Ancylostoma duodenale en Ecuador. Diagnóstico por duodenoscopia
}

\author{
Manuel Calvopiña, Jessica Flores, Isabel Guaman, Gabriela Lara y Jeyson Abarca
}

\section{Chronic and severe anemia caused by Ancylostoma duodenale in Ecuador. Diagnosis by duodenoscopy}

For 11 years, a 38-year-old male residing in a subtropical region of Ecuador, was repeatedly diagnosed with chronic anemia, and treated with blood transfusions in a hospital of province of Cotopaxi, Ecuador. He was transferred to Quito for severe anemia, having hemoglobin of $4 \mathrm{~g} / \mathrm{dL}$. Duodenoscopy was performed and adult nematodes, identified later as Ancylostoma duodenale, were observed. The patient was successfully treated with albendazole for five consecutive days and given blood transfusions. In the control visit at eight months, without anemia and no hookworm ova in the stool examined were found.

Key words: Hookworm; Ancylostoma duodenale; anemia; duodenoscopy; Ecuador.

Palabras clave: Uncinariasis; Ancylostoma duodenale; anemia, duodenoscopia; Ecuador.
Universidad De Las Américas (UDLA), Quito, Ecuador. OneHealth Group, Escuela de Medicina, Facultad de Ciencias de la Salud (MC) Hospital "Eugenio Espejo", Quito, Ecuador. Servicio de Gastroenterología y Medicina Interna (JF, IG, GL, YA).

Conflictos de interés: Ninguno. Financiamiento: Ninguno.

Recibido: 17 de marzo de 2017 Aceptado: 31 de julio de 2017

Correspondencia a: Manuel Calvopiña

manuelcalvopina@gmail.com

\section{Introducción}

L a anquilostomiasis, llamada también anemia tropical, es una parasitosis intestinal importante en todo el mundo que afecta a aproximadamente 576 millones de personas y está incluida en el grupo de las Enfermedades Tropicales Desatendidas (NTD) de la Organización Mundial de la Salud. En Latinoamérica y el Caribe, 346 millones de personas están en riesgo, con 50 millones de infectados ${ }^{1}$.

Las especies que infectan a los humanos son Ancylostoma duodenale, A. ceylanicum y Necator americanus. Los anquilostomas adultos difieren principalmente en la estructura de su abertura bucal; Ancylostoma con dos pares de dientes o ganchos de igual tamaño y Necator con un par de placas cortantes. La especie predominante en las zonas subtropicales y tropicales de la costa de Pacífico en América del Sur es $A$. duodenale ${ }^{2}$. Ambas especies se adhieren a la mucosa del intestino delgado, absorben sangre, causan erosiones, úlceras, y favorecen la pérdida de sangre por secreción de sustancias anticoagulantes y enzimas. La anemia resultante puede ser leve, moderada o grave, dependiendo de la carga parasitaria ${ }^{2}$. La anemia grave por la infección de anquilostomas se estima que causa 50.000 muertes anualmente.

Se presenta el caso de un paciente con una anemia crónica de larga data, en que durante la endoscopia se observaron numerosos nematodos en mucosa duodenal que se identificaron posteriormente como A. duodenale.

\section{Caso clínico}

Varón de 38 años de edad, nacido y residente en Moraspungo, provincia de Cotopaxi, Ecuador, analfabeto, con deficiencia mental, con historia de no tener hogar fijo, vivir en pobres condiciones higiénicas y vagar descalzo por las calles. Moraspungo se encuentra a $500 \mathrm{~m}$ sobre el nivel del mar, es montañoso, con clima subtropical, temperaturas de 22 a $24{ }^{\circ} \mathrm{C}$, lluvioso y húmedo. Fue transferido a Quito por una anemia crónica, con antecedente de transfusiones de sangre hacía 11 años. En el último año había requerido transfusiones cada tres meses. La última vez con hemoglobina de $1,8 \mathrm{mg} / \mathrm{dl}$ fue transfundido con dos paquetes de glóbulos rojos de $300 \mathrm{ml}$ cada uno.

$\mathrm{Al}$ ingresar al hospital en Quito, el paciente presentaba astenia, palidez, presión arterial de 112/70 mm/Hg, frecuencia respiratoria de 20 por min, frecuencia cardiaca de 88 por min y temperatura de $36,5^{\circ} \mathrm{C}$. Al examen físico estaba consciente, deshidratado, afebril, con escleras anictéricas y conjuntivas pálidas. A la auscultación cardíaca presentaba un soplo sistólico de eyección en el foco mitral. Se observaron heces de color oscuro. Las pruebas de laboratorio informaron una hemoglobina $4 \mathrm{mg} /$ $\mathrm{dL}$, hematocrito $12,8 \%$, VCM 81; 9.420 leucocitos $/ \mathrm{mm}^{3}$ con un diferencial de $65 \%$ neutrófilos, $32 \%$ linfocitos, $1 \%$ monocitos y $2 \%$ eosinófilos; recuento de plaquetas $213.000 \mathrm{~mm}^{3}$. Todas las pruebas bioquímicas, incluyendo electrolitos, glucosa, de función hepática, renal y tiroidea estaban dentro de los límites normales. La serología para VIH y VHB fueron negativas. El examen microscópico de heces mediante observación directa fue positivo para 
Figura 1. Duodenoscopia. Se observan varios ejemplares de nematodos adultos unidos a la mucosa duodenal. Algunos fueron extraídos del sitio donde se tomó la biopsia para la identificación de la especie.

Figura 2. Microfotografía del aparato oral de un parásito adulto obtenido en la biopsia duodenal mostrando la parte superior de un diente, característico de Ancylostoma duodenale.
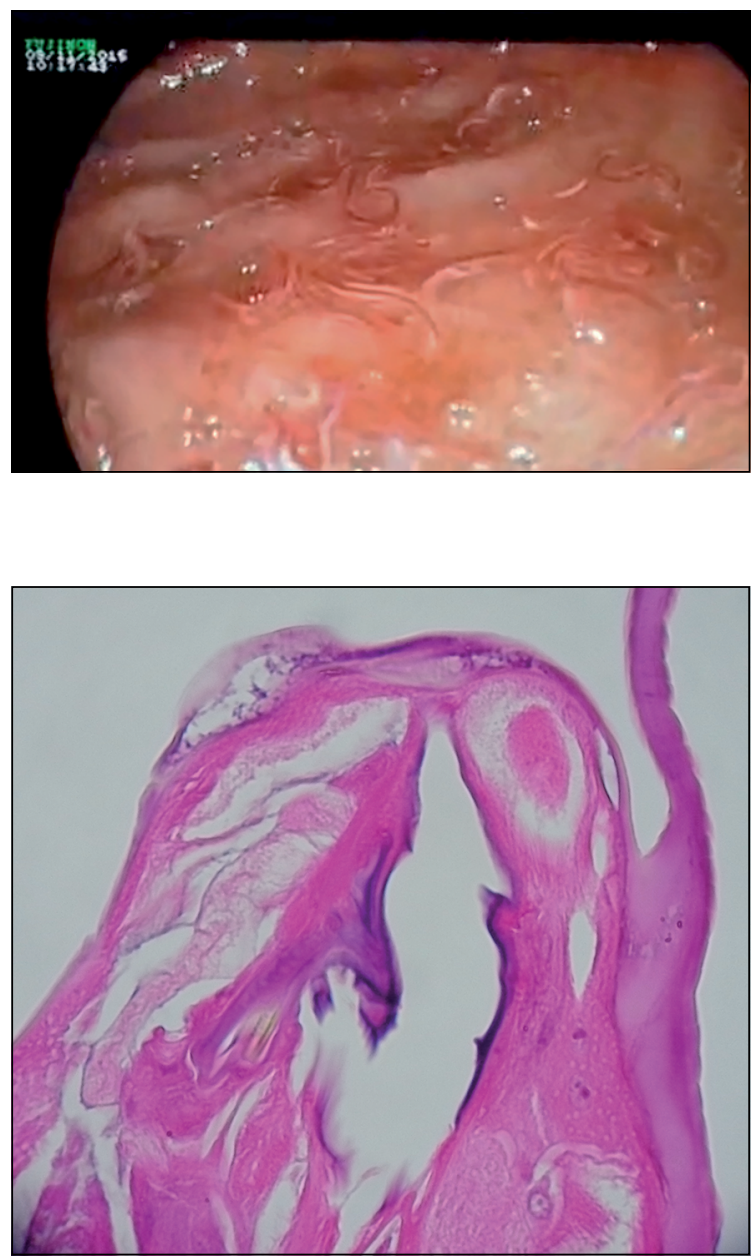

sangre oculta y quistes de Endolimax nana, sin presencia de huevos de anquilostoma. Durante la duodenoscopia se detectó una gastropatía erosiva antral; en el duodeno se observaron numerosos parásitos alargados de color nacarado, de aproximadamente $11 \mathrm{~mm}$ de longitud moviéndose activamente (Figura 1). Se realizó una biopsia de la mucosa duodenal y el análisis histopatológico de una sección longitudinal teñida con hematoxilina-eosina mostró un nematodo adulto de 11,5 mm de longitud, con dos pares de dientes en su abertura bucal, característicos de la especie $A$. duodenale (Figura 2).

El paciente fue tratado con seis paquetes globulares de $300 \mathrm{ml}$ cada uno, vitamina K, albendazol $400 \mathrm{mg} /$ día durante cinco días consecutivos, seguido de metronidazol y hierro vía oral. Después de 15 días de hospitalización, fue dado de alta en condiciones clínicas estables. En la visita de seguimiento en ocho meses, las pruebas de laboratorio mostraron una hemoglobina de $12,3 \mathrm{mg} / \mathrm{dL}$ y hematocrito $38 \%$. Utilizando un método de concentración en muestra de heces, no se encontraron huevos de anquilostomas.

\section{Discusión}

Comunicamos el caso de una anemia crónica grave causada por la infección por anquilostomas. Es importante destacar que el caso enfatiza la existencia de esta nematodiasis en Ecuador y además, la identificación de la especie infectante como $A$. duodenale. Además, es el primer caso de infección por anquilostomas diagnosticado por duodenoscopia en Ecuador, a través de la observación de parásitos adultos en el duodeno, a pesar de no encontrar huevos en las heces y la ausencia de hipereosinofilia en el recuento de leucocitos.

En el Ecuador, se ha reportado la existencia de anquilostomiasis en las regiones de la costa del Pacífico y Amazonía $^{3-9}$, con prevalencias variables dependiendo de la región geográfica. Así, en la región costera del Pacífico, $25 \%$ de afroecuatorianos residentes en el cantón Eloy Alfaro, provincia de Esmeraldas, estaban infectados ${ }^{3}$. Un estudio reciente en la misma población encontró una tasa de infección de $9,1 \%{ }^{4}$. La prevalencia en la ciudad de Portoviejo-Manabí fue de $1,7 \% \%^{5}$, igualmente en comunidades ubicadas al noroccidente de la provincia de Pichincha ${ }^{6}$. En la región amazónica, 24,1\% de los indígenas Quichua de la provincia del Napo estaban infectados ${ }^{7}$, mientras que $1,8 \%$ de los habitantes del río Paquisha en la provincia de Zamora Chinchipe ${ }^{8}$. Álvarez reportó una tasa de infección por anquilostomas de $68,1 \%$ en varias áreas de las provincias del Pacífico y Amazonía 9 . En la región andina no se ha detectado infección por anquilostomas ${ }^{10}$. Nuestro paciente provenía de un área subtropical de la región costera del Pacífico, donde la lluvia y la humedad son adecuadas para el desarrollo de larvas infecciosas de anquilostomas y otros nematodos.

Los factores de riesgo asociados con la infección por anquilostomas incluyen vivir en zonas rurales tropicales o subtropicales, bajo nivel socioeconómico, mala higiene y caminar descalzo. Todos estos factores estuvieron presentes en el caso presentado. La deficiencia mental, el analfabetismo y la deambulación podrían añadirse como otros factores de riesgo a considerar.

Las infecciones por helmintos, incluyendo la infección por anquilostomas, generalmente inducen hipereosinofi$\operatorname{lia}^{11,12}$. En este caso el paciente no la presentó. La ausencia de hipereosinofilia no descarta el diagnóstico, ya que se han reportado otros casos sin hipereosinofilia, lo que puede estar asociado con el reclutamiento de eosinófilos en los tejidos ${ }^{13}$.

El diagnóstico de infección por anquilostomas se realiza generalmente observando sus huevos característicos en las heces, aunque la sensibilidad de la detección puede variar dependiendo de la metodología empleada ${ }^{3}$. Las muestras fecales pueden ser negativas, aunque se observan parásitos adultos en procedimientos endoscópicos ${ }^{11-13}$. Este caso de infección por anquilostomas fue diagnos- 
ticada por duodenoscopia, a través de la observación de parásitos adultos en el duodeno, siendo el primer caso comunicado en Ecuador, aunque reportado en otros países $^{11,13,14}$. Es interesante enfatizar que no se observaron huevos de anquilostomas en el examen directo de heces, como lo hicieron en casos en Taiwán y China ${ }^{12-14}$. Un único examen directo de heces tiene una sensibilidad baja, por eso se recomienda el análisis de tres muestras durante tres días consecutivos o con el uso de métodos de concentración. En Ecuador, los laboratorios del Ministerio de Salud Pública (MSP) no ofrecen ningún método de concentración. Debido a que los métodos serológicos pueden dar una reacción cruzada con otros parásitos del orden Strongyloidea no se recomiendan y no están disponibles en Ecuador.

El tratamiento recomendado en las anquilostomiasis es la administración oral de albendazol en dosis única de 400 $\mathrm{mg}$. Sin embargo, varios estudios han reportado fracasos de tratamiento con dosis única, con tasas de curación entre $61 \%$ y $98 \%$, por lo que se recomienda administrar 400 mg de albendazol durante tres días consecutivos o como dosis única de $800 \mathrm{mg}$. En nuestro paciente, la eliminación completa de $A$. duodenale se obtuvo administrando albendazol $400 \mathrm{mg} /$ día durante cinco días. Mebendazol, pamoato de pirantel e ivermectina se consideran fárma- cos de segunda elección ${ }^{15}$. Sin embargo, en Ecuador, la distribución masiva de ivermectina después de 17 años no ha tenido impacto en la reducción de la infección por anquilostomas ${ }^{4}$.

Agradecimientos. Al paciente y a su madre por permitir comunicar este caso clínico y al personal médico y de laboratorio del Hospital "Eugenio Espejo" por facilitar los resultados de laboratorio.

\section{Resumen}

Durante 11 años un varón de 38 años de edad, residente en una región subtropical de Ecuador, había sido diagnosticado de anemia crónica y tratado con transfusiones de sangre en un hospital de la provincia de Cotopaxi, Ecuador. Fue transferido a Quito por una anemia grave, con hemoglobina de $4 \mathrm{~g} / \mathrm{dL}$. Se realizó una duodenoscopia en que se observaron nemátodos adultos, identificados posteriormente como Ancylostoma duodenale. El paciente fue tratado exitosamente con albendazol durante cinco días consecutivos y transfusiones de sangre. En el seguimiento a los ocho meses, no se encontró anemia ni huevos de anquilostomas en el examen de heces.

\section{Referencias bibliográficas}

1.- Hotez P J, Bottazzi M E, Franco-Paredes C, Ault S K, Periago M R. The neglected tropical diseases of Latin America and the Caribbean: a review of disease burden and distribution and a roadmap for control and elimination. PLoS Negl Trop Dis 2008; 2: e300.

2.- Brown H W, Neva F A. Hookworm. Basic Clinical Parasitology, 5th edition. 1983, Norwalk, Conn: Appleton-Century-Crofts, Connecticut, Unites States of America; pág 122-3.

3.- Cooper P J, Guevara A, Guderian R H. Intestinal helminthiases in Ecuador: the relationship between prevalence, genetic, and socioeconomic factors. Rev Soc Bras Med Trop 1993; 26: 175-80.

4.- Moncayo A L, Vaca M, Amorim L, Rodríguez A, Erazo S, Oviedo G, et al. Impact of long-term treatment with ivermectin on the prevalence and intensity of soiltransmitted helminth infections. PLoS Negl Trop Dis 2008; 2: e293.

5.- Andrade C, Alava T, De Palacio I A, Del Poggio P, Jamoletti C, Gulletta M, et al.
Prevalence and intensity of soil-transmitted helminthiasis in the city of Portoviejo (Ecuador). Mem Inst Oswaldo Cruz 2001; 96: 1075-9.

6.- $\quad$ Sackey M E, Weigel M M, Armijos R X. Predictors and nutritional consequences of intestinal parasitic infections in rural Ecuadorian children. J Trop Pediatr 2003; 49: 17-23.

7.- San Sebastián M, Santi S. Control of intestinal helminths in schoolchildren in Low-Napo, Ecuador: impact of a two-year chemotherapy program. Rev Soc Bras Med Trop 2000; 33 : 69-73.

8.- González M V, Flores S A, Cruz-Erazo C, Sánchez D E. Prevalencia de geohelmintos y factores socioambientales en zonas urbanas y rurales, cantón Paquisha, Ecuador. CEDAMAZ 2014; 4: 4-13.

9.- Álvarez J. El parasitismo intestinal en el Ecuador. Rev Ed Med Cont Schering 1976; 8: 12-17.

10.- Jacobsen K H, Ribeiro P S, Quist B K, Rydbeck B V. Prevalence of intestinal parasites in young Quichua children in the highlands of rural Ecuador. J Health Popul
Nutr 2007; 25: 399-405

11.- García-Compean D, Jáquez-Quintana J O, González-González J A, Maldonado-Garza H J. Midgut bleeding due to uncinariasis and diagnosed by capsule endoscopy: a clinical case. Rev Gastroenterol Mex 2013; 78: 196-7.

12.- Wang C H, Lee S C, Huang S S, Chang L C. Hookworm infection in a healthy adult that manifested as severe eosinophilia and diarrhea. J Microbiol Immunol Infect 2011; 44: 484-7.

13.- Wu K-L, Chuah S-K, Hsu C-Ch, Chiu K-W, Chiu Y-Ch, Changchien Ch-S. Endoscopic diagnosis of hookworm. A case report. World J Gastroenterol 2013; 19: 3915-21.

14.- Hyun H J, Kim E M, Park S Y, Jung J O, Chai J Y, Hong S T. A case of severe anemia by Necator americanus infection in Korea. J Korean Med Sci 2010; 25: 1802-4.

15.- Krepel H P, Haring T, Baeta S, Polderman A M. Treatment of mixed Oesophagostomum and hookworm infection: effect of albendazole, pyrantel pamoate, levamisole and thiabendazole. Trans R Soc Trop Med Hyg 1993; 87: 87-9. 